$\left\{\begin{array}{l}\text { JOURNAL OF ETHNOBIOLOGY } \\ \text { AND ETHNOMEDICINE }\end{array}\right.$

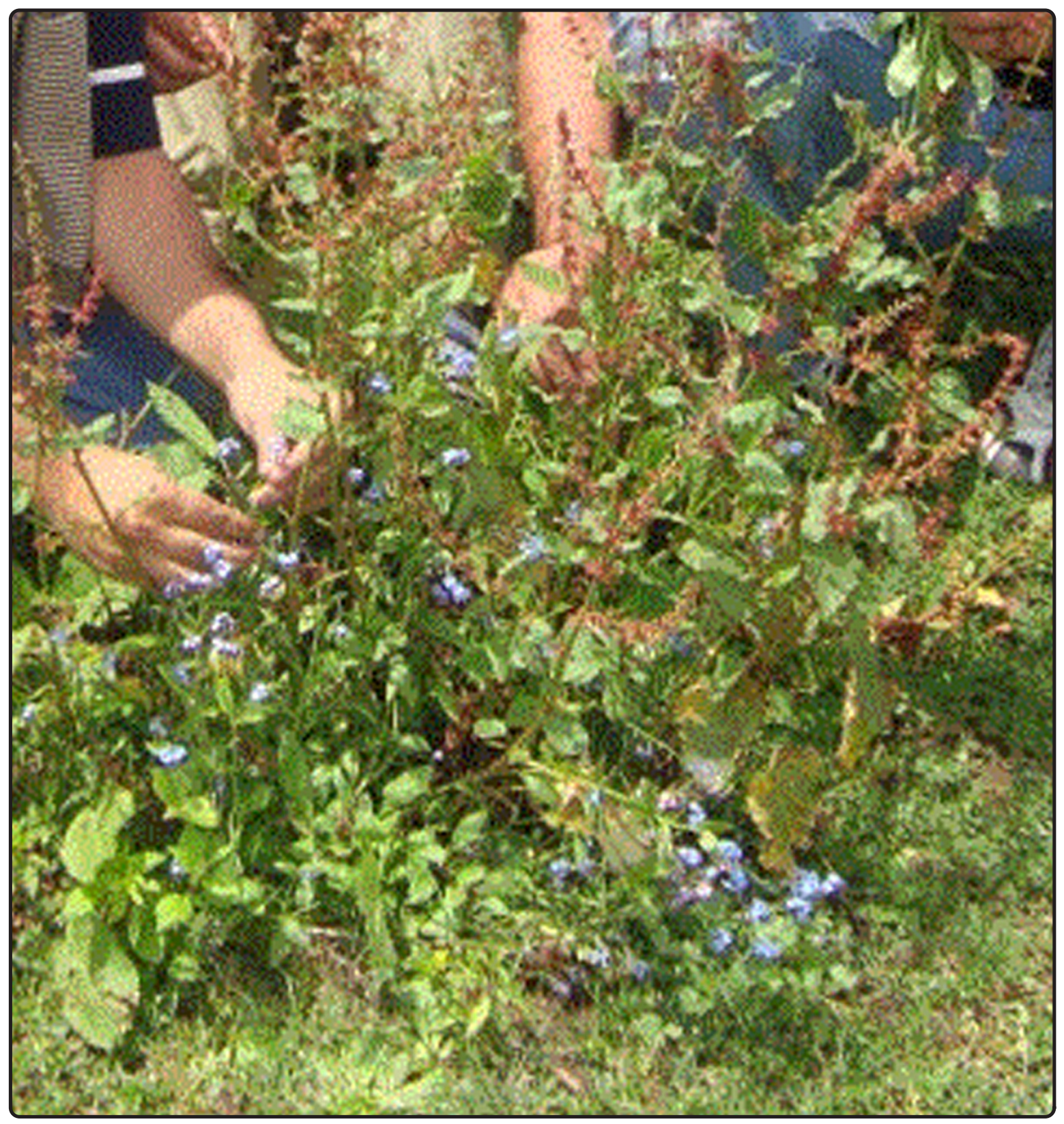

\title{
Quantitative ethnomedicinal study of plants used in the skardu valley at high altitude of Karakoram-Himalayan range, Pakistan
}

Bano et al. 


\title{
Quantitative ethnomedicinal study of plants used in the skardu valley at high altitude of Karakoram-Himalayan range, Pakistan
}

\author{
Abida Bano ${ }^{1}$, Mushtaq Ahmad ${ }^{1,2}$, Taibi Ben Hadda ${ }^{3 *}$, Abdul Saboor ${ }^{4}$, Shazia Sultana ${ }^{1,2}$, Muhammad Zafar ${ }^{1}$, \\ Muhammad Pukhtoon Zada Khan ${ }^{1}$, Muhammad Arshad ${ }^{5}$ and Muhammad Aqeel Ashraf ${ }^{6}$
}

\begin{abstract}
Background: The tribal inhabitants of the Skardu valley (Pakistan) live in an area of great endemic botanic diversity. This paper presents the first quantitative ethnomedicinal spectrum of the valley and information on the uses of medicinal plant. This paper aims to analyze and catalogue such knowledge based on Relative Frequency Citation (RFC) and Use Value (UV) of medicinal plants in addition to the configuration of the Pearson correlation coefficient.

Methods: The field study was carried out over a period of approximately 2 years (2011-2013) using semi-structured interviews with 71 informants (most of the informants belonged to an age between 50 and 70 years) in six remote locations in the valley. Ethnomedicinal data was analyzed using frequency citation (FC), relative frequency of citation (RFC) and use value (UV) along with a Pearson correlation coefficient (PCC). Demographic characteristics of participants, ethnobotanical inventory of plants and data on medicinal application and administration were recorded.

Results: A total of 50 medicinal plants belonging to 25 families were reported to be used against 33 different ailments in the valley. The maximum reported medicinal plant families were Asteraceae (7 report species), Lamiaceae (6) , Polygonaceae (4) and Rosaceae (4), the most dominant life form of the species includes herbs (38) followed by shrubs and subshrubs (12), the most frequent used part was leaves (41\%) followed by root (26\%), flower (14\%), fruit (9\%), seeds (8\%), bulb (1\%) and bark (1\%), the most common preparation and administration methods were infusion (32\%), decoction (26\%), paste (18\%), herbal juice (17\%) and powder drug (7\%). The Pearson correlation coefficient between RFC and UV was 0.732 showing highly positive significant association.

Conclusions: In this study, we have documented considerable indigenous knowledge about the native medicinal plants in Skardu valley for treating common ailments which are ready to be further investigated phytochemically and pharmacologically which leads to natural drug discovery development. The study has various socioeconomic dimensions which are associated with the local communities.
\end{abstract}

Keywords: Quantitative ethnobotany, Medicinal plants, Skardu valley, Northern Pakistan

\section{Background}

Pakistan is rich in diversity of medicinal and aromatic flora due to its unique phytogeography with diverse climatic conditions. About 400-600 medicinal plant species out of 5700 are estimated to exist in Pakistan. In early 1950 almost $84 \%$ of the Pakistani population mainly dependent on traditional medicines as a primary

\footnotetext{
* Correspondence: taibi.ben.hadda@gmail.com

${ }^{3}$ Laboratoire de Chimie des Matériaux, Faculté des Sciences, Université Mohammed Premier, Oujda 60000, Morocco

Full list of author information is available at the end of the article
}

health care source [1] but nowadays this dependency is limited only to the remote areas due to rapid change in lifestyle and modernization [2]. However, the Northern Pakistan is still considered to be one of the richest regions in the country in terms of its biodiversity and culture of utilization of unique medicinal plant resources [3]. The three mountain ranges, the Himalaya, the Karakorum and the Hindu Kush (HKH) collectively contain about 25,000 species (about $10 \%$ of world plant species), out of which around 10,000 are economically or medicinally valuable [4]. These mountainous regions provide a naturally conducive 
environment for the growth of medicinal flora. People living in these regions of Pakistan are using plants in many ways like medicines, timber wood, firewood, food and fodder [5]. In Himalayan ranges about $70 \%$ of the medicinal flora and animals are used as wild species, $70-80 \%$ of the inhabitants are dependent on traditional medicines using plants to cure their common ailments [6]. These regions are rich with endemic species and around 1,000 species of vascular plants are known to occur in the northern mountain regions of Pakistan [7-9]. Alam [10] reported eight endemic species from Gilgit Baltistan, out of these Astragalus clarkeanus, Asperula oppositifolia subsp. baltistanica, Berberis pseudoumbellata subsp. gilgitica, Haplophyllum gilesii and Tanacetum baltistanicum are found critically endangered (CR) while Aconitum violaceum var. weileri and Rhodiola saxifragoides are vulnerable (VU).

Generally in Deosai plateau, about 342 species of plants belonging to 36 families and 142 genera have been recorded in the flora of Pakistan so far, while to the best of our knowledge, the number of species used as medicinal are not systematically recorded in literature. This high level of biodiversity on the plateau is due to several reasons, including topography, location of the plateau (Junction of major mountain ranges) and local adaptation of its plant and animal species [11]. Sultana et al. [12] reported 43 species of Poaceae, 32 species of Cyperaceae and 4 species of Juncaceae from Deosai plateau. A total of 114 plant species belonging to 28 families were found around the Sheosar Lake of Deosai plateau [13]. These studies on Deosai $[11,12,13]$ were based on biodiversity, altitudinal distribution of species and phytosociological expeditions. The present study focuses only on medicinal plant species frequently used by the local populace of Skardu valley. The study area has a rich potential for utilization and consumption of medicinal and aromatic plants. These areas are also the potential sites for exporting tradable medicinal plants on a sustainable basis [14].

Numbers of ethnobotanical studies on the use of medicinal plants in traditional health care were carried out in the neighboring parts of these ranges by previous ethnobotanists [14-20]. Whereas, Skardu valley was neglected all the time, due to its rough geography, high altitude and strict cultural and religious bans for outsiders to document such source of knowledge. There is a typical social and economic fabric of this region which is highly associated to the flora and fauna available in the valley. People give much importance to the plants and trees for their domestic and medicinal uses. An in depth study has not been pursued in the past. To fill up this gap, the folk medicinal uses of the plants of the valley were documented in the form of an ethnomedicinal inventory. Specifically the main aims of this investigation are: (i) to compile the ethnoflora with traditional medicinal applications of the Skardu valley for the scientific community, (ii) to assess quantitatively the ethnomedicinal data using RFC and UV indices in order to look for most cited and used species which have not been previously reported or with limited past reports on medicinal uses that may provide baseline data for future evaluation regarding their pharmacological and clinical screening.

\section{Materials and methods}

\section{An overview of the geography and climate of the} study area

The Karakoram Range covers the borders of India, China and Pakistan (in the regions of Gilgit-Baltistan). The Himalaya range in Pakistan occupies the regions of Deosai, Chilas, Kaghan, Kohistan and Kashmir. Many of the peaks of this range are above $8000 \mathrm{~m}$ as Godwin Austin/K-2 (8611 m), Nanga Parbat (8126 m), Gasherbrum I (8080 m), Broad Peak (8051 m) and Gasherbrum II $(8035 \mathrm{~m})$. Outside the Arctic, world's largest glacier, Siachin (78 km long), is located here with temperature close to $-50^{\circ} \mathrm{C}$. Skardu valley is located at $35^{\circ} 18^{\prime}$ north latitude and $75^{\circ} 37^{\prime}$ east longitude in Baltistan region, Northern Pakistan. Temperatures in the base of valley range from nearly $38^{\circ} \mathrm{C}$ in summer to less than $-25^{\circ} \mathrm{C}$ in winter. Skardu borders District Ghanche to the east, Indian-occupied Kashmir to the south, the Chinese province of Sinkiang to the north and the Deosai Plateau to the southwest. The Deosai Plateau $\left(30^{\circ} 00\right.$ N $\left.75^{\circ} 30 \mathrm{E}\right)$ is located in the north of the main Himalayan range. Its height is about 4,115 $\mathrm{m}$ (14,500 feet) above the timberline and is considered to be one of the highest plateaus of the world. For over half the year (between September - May), Deosai is snow covered and inaccessible to visit (snow is 7-8 yard deep). Deosai is accessible from Skardu in the north and the Astore valley in the west. It is about $30 \mathrm{~km}$ from Skardu and covers an area of almost $5,000 \mathrm{~km}^{2}$. The various sites of Deosai Plateau are Shatun Nala, Bara Pani, Kala Pani, Murtaza Camp, Burzil Top, Shausar Lake, Gultari, Chota Deosai, Barila, Chilam Chauki, Farenshot and Dumbo Bahao. This ethnomedicinal exploration was confined to nine botanically rich sites of the valley includes Kachura, Hoto, Shigari, Sadpara Lake, Mehdi Abad and four selected sites of Deosai plateau (Shausar lake, Bara pani, Kala pani, Gultari) (Figure 1). As our focus is on the most frequently used medicinal plants, the local herbalist and laymen having rich traditional knowledge preferred these sites for medicinal plant collection. Elevations of the selected study sites range from approximately 2,270 $\mathrm{m}$ to $4,115 \mathrm{~m}$ on the Deosai Plains. The flora of the valley is rich and diverse due to its altitude, unique climate and other topographic conditions [21]. 

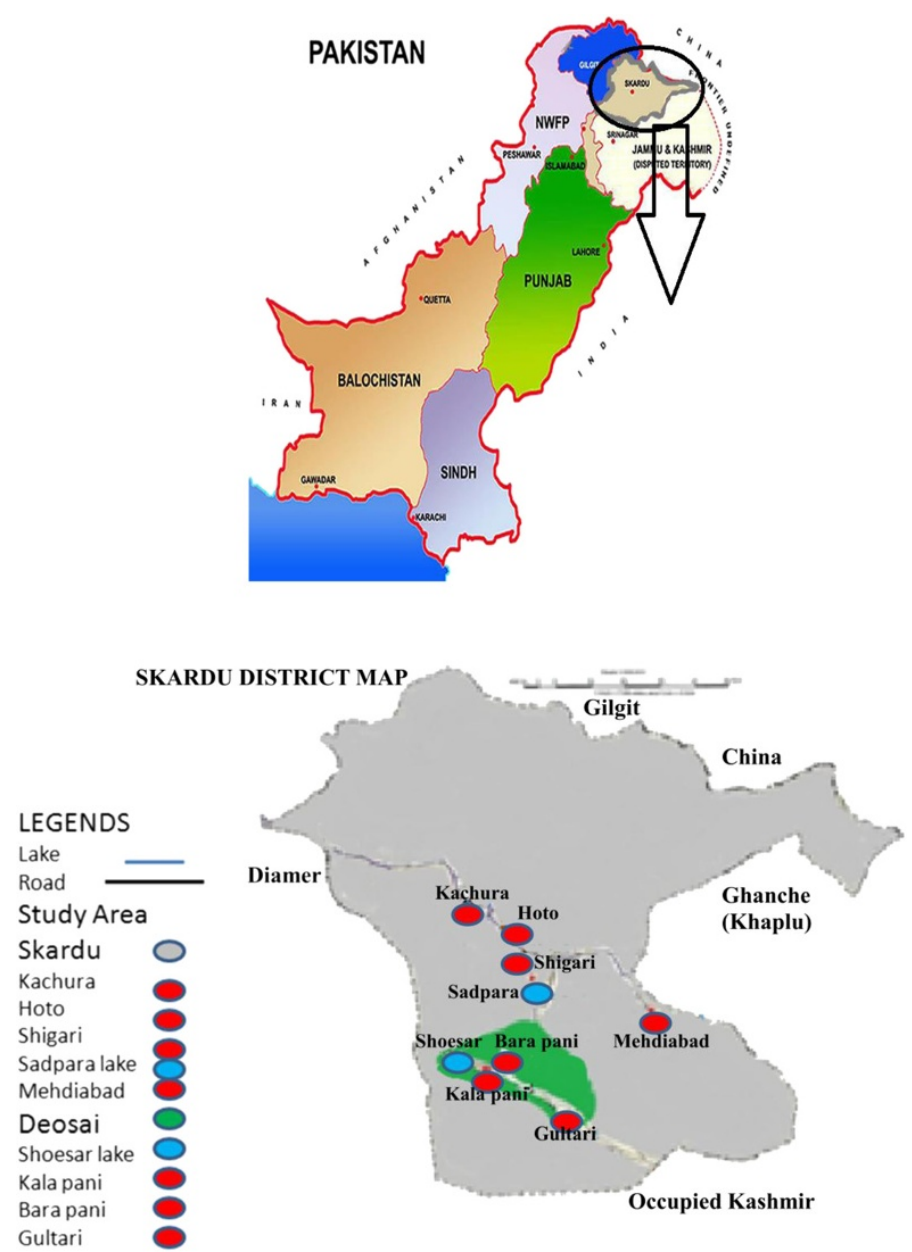

Figure 1 Map of District Skardu showing the study sites.

\section{Ethnomedicinal data collection and ethnographic composition}

Field work consisted of data documentation, plant collection and photography. Prior rural appraisal (PRA) approach was adopted according to Kyoto protocol concerning the intellectual property rights of local inhabitants and plant resources of the area. Formal written consent, including consent for publication was received from all the informants before the interviews began. The method employed during the study was designed with the sole purpose for eliciting the precious wealth of local knowledge on medicinal plant use [22]. A total of 71 informants with a different age range were randomly selected for interviews. A total of 35 men (49\%), 30 women $(42 \%)$ and men traditional healers $11(15 \%)$ were interviewed. The informants were divided into two age groups (1) 41-60 and (2) 61-80 years old. Most of the informants belonged to an age between 50 and 70 years. The ethnic composition of the valley is quite diverse, mainly resides with Baltistan Mongle, Mon, Hor,
Brokpa and Kashmiri. Primary local language spoken in the area is Balti ( $90 \%$ of the population) while the other locally known languages include Shina, Burshishki and Wakhi.

The selection of informants was mainly based on their rich indigenous knowledge and long term experience of utilization of plants. Field surveys mainly comprised, general meeting, male interviews, female interviews, local herbalists (Hakims) interviews and transect walk. The semi-structured questionnaire based interviews were begun with informants after explaining the aims of the study. The informants were asked various questions about their traditional knowledge, plant use, disease treated, part used and the method of preparation and administration. Interviews were generally conducted in the local language of Balti at village male gathering places, mosques and sometimes in houses. In order to collect detailed information relating to herbal medicine inhabitants of the community were requested to share the knowledge of medicinal plant utilization in local language. All interviews 
were conducted with the assistance of native translators in their local language. All documented data were then translated into English.

Data on local names of the plants, part used, method of preparation, administration route, and application to treat diseases was recorded. In some cases field photos of the medicinal plants were used to confirm the local names by the informants. The plant specimens were collected during field survey with the help of informants. The medicinal plants were identified using regional Flora [8] and plant taxonomists at the Herbarium of Pakistan (ISL) Quaid-i-Azam University.

\section{Socio-economic conditions of Skardu valley}

The flora of the Skardu valley is very diverse with hundreds of medicinal plants with useful pharmaceutical values and a number of economically important species of wild plants. Biodiversity is a significant natural resource for the socio-economic welfare of the people of the valley. Wild plant species and traditional medicine were one of the economical sources for the local communities. Animal and plant communities supported the development of early populaces of this region for centuries, providing the base for the evolution from hunting to forestry, agriculture, animal husbandry and now tourism and trade. The narrow valley and high mountains kept Skardu physically isolated which forced indigenous people to depend on local biodiversity for food and other essential needs. Continuing use and maintenance of biological diversity is important to the people of Skardu. Economic development of Skardu valley depends on management of high diversity of crops, maintaining high pastures, raising fodder species in varied mountain environment and development of livestock biodiversity and medicinal plants. These will be the feasible options for ensuring food security and generating cash income of the people of the valley.

\section{Quantitative ethnomedicinal data analysis Relative Frequency Citation (RFC)}

The collected ethnomedicinal information was quantitatively analyzed using an index of relative frequency citation (RFC) as;

$$
\mathrm{RFC}=\mathrm{FC} / \mathrm{N}(0<\mathrm{RFC}<1)
$$

This index shows the local importance of each species and it is given by the frequency of citation (FC, the number of informants mentioning the use of the species) divided by the total number of informants participating in the survey $(\mathrm{N})$, without considering the use-categories [23].

\section{Use Value (UV)}

The Use Value (UV) demonstrates the relative importance of plants known locally. It was calculated using the following formula [24].

$$
\mathrm{UV}=\sum \mathrm{Ui} / \mathrm{N}
$$

Where $\mathrm{Ui}$ is the number of uses mentioned by each informant for a given species and $\mathrm{N}$ is the total number of informants.

\section{Pearson correlation coefficient}

Pearson product-moment correlation coefficient is a good measure to numerically quantify the nature of the linear relationship between two variables. Pearson's correlation coefficient is the ratio of the covariance between two variables to their standard deviations and calculated from a sample as

$$
r=\frac{\operatorname{COV}(X, Y)}{S D(X) * S D(Y)}=\frac{E(X-\bar{X})(Y-\bar{Y})}{S D(X) * S D(Y)}
$$

Where $\mathrm{r}$ is the Pearson correlation coefficient for the given sample, $\mathrm{COV}$ is the covariance, $\mathrm{X}$ and $\mathrm{Y}$ are the variables for which we are interested to explore the relationships and SD is the standard deviation for the same variables and calculated as

$$
\begin{aligned}
& S D(X)=\sqrt{\frac{1}{n-1} \sum_{i=1}^{n}\left(X_{i}-\bar{X}\right)^{2}} \\
& \bar{X}=\frac{\sum_{i=1}^{n} X_{i}}{n}
\end{aligned}
$$

Where $\mathrm{X}$ bar is the mean value of $\mathrm{X}$ and $\mathrm{n}$ is the sample size [25]. Similarly $\mathrm{SD}(\mathrm{Y})$ can be estimated. To test the null hypothesis of no linear relationship between the variables $(\rho=0)$, z-test was used at the 5 percent level of significance. The value of z-stat can be obtained by

$$
z=\frac{\sqrt{n-3}}{2} \ln \left[\frac{(1+r)(1-\rho)}{(1-r)(1+\rho)}\right]
$$

Where $\rho$ is the population coefficient. If the P - value of $\mathrm{z}$-stat is less than 5 percent, it means the two variables have a significant linear association with each other [26]. In our case the two variables of interest are the RFC and UV. Since the correlation is the quantitative measure of the degree to which patterns RFV and UV respond while RFC vary across different species as do UV. The correlation squared (r2) measures the cross species variability in RFC that is explained by the variance in UV and is obtained simply by taking the square of the $\mathrm{r}$ [27]. 


\section{Table 1 Use reports}

Botanical name and voucher number

Allium humile Kunth

Use reports

SK 103

Carum carvi L.

SK 104

Heracleum candicans Wall. ex DC.

SK 105

Artemisia sieversiana Ehrh.

SK 108

Cichorium intybus L.

SK 109

Jurinea dolomiaea Boiss

SK 110

Pseudognaphalium luteoalbum (L.) Hilliard \& B. L. Burtt.

SK 111

Senecio chrysanthemoides DC.

SK 112

Seriphidium brevifolium ( Wall. ex DC. ) Ling \& Y.R.Ling

SK 107

Tanacetum gracile Hook. f. \& Thoms.

SK 113

Berberis vulgaris $\mathrm{L}$.

SK114

Lepidium latifolium L.

SK 115

Codonopsis clematidae C.B. Clarke

SK 116

Capparis spinosa $\mathrm{L}$.

SK 117

Chenopodium album L.

SK 118

Chenopodium botrys L.

SK 119

Rhodiola imbricata Edgew.

SK 120

Juniperus excelsa M. Bieb.

SK 121

Hippophae rhamnoides L.

SK 122

Ephedra gerardiana Wall.

SK 123

Swertia petiolata Royle

SK 124

Gentiana olivieri Griseb.

SK 125
Table 1 Use reports (Continued)

Geranium nepalense Sweet

SK 126

Dracocephalum nuristanicum Rech. f. \& Edelb.

SK 106

Mentha royleana Wall. ex Benth

SK 127

Perovskia abrotanoides Kar.

SK 128

Nepeta leucolaena Benth. ex Hook.f

SK 129

Thymus linearis Benth.

SK 130

Prunella vulgaris $\mathrm{L}$.

SK 131

Rhododendron anthopogon D. Don.

SK 132

Epilobium latifolium L.

SK 133

Astragalus psilocentros Fisch

SK 134

Sophora mollis Graham

SK 135

Plantago lanceolata $\mathrm{L}$.

SK 136

Bistorta amplexicaulis (D.Don.) Greene

SK 137

Oxyria digyna (L.) Hill.

3

SK 138

Polygonum affine D. Don.

SK 139

Rheum australe D. Don

6

SK 140

Primula denticulata Smith

SK 141

Aconitum violaceum Jacquem. ex Stapf

SK 142

Caltha alba Jacquem.

SK 143

Delphinium brunonianum Royle

4

SK 144

Potentilla argyrophylla Wall. ex Lehm. var. atrosanguinea (G.Lodd.) Hook.f.

SK 145

Potentilla bifurca $\mathrm{L}$.

1

SK 146 
Table 1 Use reports (Continued)

\begin{tabular}{ll}
\hline Potentilla salesoviana Steph. & 4 \\
SK 152 & 1 \\
Rosa brunonii Lindl. & \\
SK 147 & 6 \\
Bergenia stracheyi (Hook. f. \& Thorns.) Engl. & \\
SK 148 & 5 \\
Bergenia ciliata (Haw.) Sternb. & \\
SK 149 & 2 \\
Pedicularis cheilanthifolia Schrenk & \\
SK 150 & 2 \\
Pedicularis pectinatiformis Bonati & \\
SK 151 & \\
\hline
\end{tabular}

\section{Ethical approval}

This ethnomedicinal study was duly approved by the ethical committees of the Herbarium (ISL), Department of Plant Sciences Quaid-i-Azam University, Islamabad and Biodiversity Action Plan (BAP-2010-2020) for Pakistan. Prior permission was sought from the Forest Department, Gilgit-Balistan Region for the collection of plants and ethnomedicinal data in the area. PIC was obtained from the informants after elaborating the aims of the survey Table 1.

\section{Results and discussion}

\section{Medicinal plant diversity, part used and administration} methods

A total of 50 plant species belonging to 44 genera of 25 families were catalogued in Table 2, with traditional uses as herbal medicine against various diseases. The study was carried out in nine selected sites of the valley to document the traditional knowledge of local people on medicinal plants. The most encountered medicinal plant families were Asteraceae (7 reported species), Lamiaceae (6), Polygonaceae (4) and Rosaceae (4) while the rest of the family was represented with variable number of less than 4 species. Our findings regarding the predominance utilization of family Asteraceae and Lamiaceae in the Skardu valley were in agreement with other ethnic-floras [28-31]. The reasons for high degree of ethnomedicinal plants of families Asteraceae and Lamiaceae in our region are due to their wide occurrence with a number of traditional uses known by the local informants. The most dominant life form of the species reported includes herbs (38) followed by shrubs and subshrubs (12). A similar pattern of life form was reported in ethnobotanical survey of our neighboring country India [32]. The parts of the plant primarily used are the leaves (41\%), while roots $(26 \%)$, flowers $(14 \%)$, fruits $(9 \%)$, seeds $(8 \%)$, bulbs and barks (1\%) are also frequently used (Figure 2).
These reports were in agreement with previous studies conducted in different parts of the world, where the leaves are cited as commonly used parts of the medicinal plants. Medicinal plants used in folk herbal remedies are prepared and administered in various forms in the valley. The most common preparation and administration methods were categorized into infusion (32\%), decoction $(26 \%)$, paste $(18 \%)$, herbal juice $(17 \%)$ and powdered drug (7\%) (Figure 3). However, these results are different in comparison with previous ethnobotanical studies $[33,34]$. The percentage of oral administration $(77 \%)$ of herbal preparation is almost higher than external or topical (23\%) application. Similar to our findings regarding the administration route of herbal drugs, the oral administration was also reported in previous work conducted in Bolivia [35]. The most treated illnesses of the Skardu valley using a number of medicinal plants are grouped into 33 pathological disorders. We found the highest number of plant species are used against stomachache ( 9 species) followed by cold/fever ( 8 species), rheumatism (7 species), wounds (6 species), Asthma/ breathing problem/difficulty in breathing (6 species), sore throat (5 species), diarrhea (4 species), eye diseases ( 3 species), hepatitis and diabetes ( 2 species each) (Figure 4). While some other treated diseases includes dysentery, pneumonia, respiratory disorders, jaundice, renal disease, lung disorders, liver disease etc. The local herbalist/Hakims (Person with indigenous knowledge on diseases, its diagnosis and folk treatment) diagnose ailments by their signs and symptoms rather than specific laboratory tests, as this knowledge travel through generations. Figure 4 shows the most treated disease using plants in this study include stomach disorder followed by cold \& fever. The findings regarding number of plants used against each disease category were dissimilar to previous work in our neighboring regions [36]. To the best of our knowledge, this variation in comparison to previous studies is additional and novel ethnomedicinal information reported from this valley. This valuable information would create a sense of social and economic ownership of unique floral resources which can help in the preservation of important plant species. By realizing the importance of their natural resources, they can provide a recreational look to the attraction of tourists in the region.

\section{Data on quantitative ethnomedicinal uses}

Quantitative value indices were calculated in this study to analyze the ethnomedicinal information. Figure 5 show the 17 most cited plants known by a majority of the informants for medicinal uses. The highest value of RFC ranked the Hippophae rhamnoides (0.91) first, followed by Rosa brunonii (0.88) and Sophora mollis $(0.85)$ as second and third respectively. These positions 
Table 2 Medicinal plants used to treat various diseases in Skardu valley

\begin{tabular}{|c|c|c|c|c|c|c|c|c|c|c|}
\hline Family & Botanical name and voucher number & Local name & Life form & Parts used/ Formulation & Applications & Administration route & *FC & **RFC & $* * * \Sigma U i$ & $* * * * U V$ \\
\hline \multirow[t]{4}{*}{ Alliaceae } & Allium humile Kunth SK 103 & Chung & Herb & Bulb infusion & $\begin{array}{l}\text { Asthma/breathing } \\
\text { problem }\end{array}$ & Oral & 13 & 0.18 & 27 & 0.38 \\
\hline & & & & & Stomach disease & & & & & \\
\hline & & & & & Jaundice & & & & & \\
\hline & & & & & Cough, cold & & & & & \\
\hline \multirow[t]{3}{*}{ Apiaceae } & Carum carvi L. SK 104 & Zera & Herb & Seed decoction & Gastric problems & Oral & 21 & 0.29 & 45 & 0.63 \\
\hline & & & & & Sore throat & & & & & \\
\hline & & & & & Rheumatism & & & & & \\
\hline \multirow[t]{2}{*}{ Apiaceae } & Heracleum candicans Wall. ex DC. SK 105 & Ghang & Herb & Leaves decoction & Leucoderma & Oral & 14 & 0.19 & 29 & 0.40 \\
\hline & & & & & Psoriasis & & & & & \\
\hline \multirow[t]{3}{*}{ Asteraceae } & Artemisia sieversiana Ehrh. SK 108 & Khampa & Subshrub & Flower infusion & Pneumonia & Oral & 33 & 0.46 & 81 & 1.14 \\
\hline & & & & Leaves decoction & Joints & Oral & & & & \\
\hline & & & & Root paste & Boils & Topical & & & & \\
\hline \multirow[t]{3}{*}{ Asteraceae } & Cichorium intybus L. SK 109 & Shantha & Herb & Root infusion & Fever & Oral & 22 & 0.30 & 54 & 0.76 \\
\hline & & & & Leaves juice & Release of gall stones & Oral & & & & \\
\hline & & & & & $\begin{array}{l}\text { Gastrointestinal } \\
\text { problems }\end{array}$ & & & & & \\
\hline \multirow[t]{4}{*}{ Asteraceae } & Jurinea dolomiaea Boiss SK 110 & Sathing & Herb & Root decoction & Cardiac & Oral & 7 & 0.09 & 9 & 0.12 \\
\hline & & & & Leaves juice & Colic & Oral & & & & \\
\hline & & & & Root poultice & Fever & Topical & & & & \\
\hline & & & & & Eruptions & & & & & \\
\hline Asteraceae & $\begin{array}{l}\text { Pseudognaphalium luteoalbum (L.) Hilliard \& } \\
\text { B. L. Burtt. SK } 111\end{array}$ & Thliri & Herb & Leaves juice & $\begin{array}{l}\text { Asthma/breathing } \\
\text { problem }\end{array}$ & Oral & 14 & 0.19 & 36 & 0.50 \\
\hline \multirow[t]{3}{*}{ Asteraceae } & Senecio chrysanthemoides DC. SK 112 & Api mindoq & Herb & Leaves decoction & Sore throat & Oral & 15 & 0.21 & 31 & 0.43 \\
\hline & & & & Flower poultice & Wounds & Topical & & & & \\
\hline & & & & Root powder & Rheumatic pain & Topical & & & & \\
\hline Asteraceae & $\begin{array}{l}\text { Seriphidium brevifolium ( Wall. ex DC. ) Ling \& } \\
\text { Y.R.Ling SK } 107\end{array}$ & Bursay & Subshrub & Flower powder & Expel worms & Oral with water & 27 & 0.38 & 63 & 0.88 \\
\hline \multirow[t]{2}{*}{ Asteraceae } & Tanacetum gracile Hook. f. \& Thoms. SK 113 & Serfo bursay & Herb & Leaves decoction & Intestinal worms & Oral & 9 & 0.12 & 19 & 0.26 \\
\hline & & & & Leaves powder & Obesity & & & & & \\
\hline \multirow[t]{3}{*}{ Berberidaceae } & Berberis vulgaris L. SK114 & Shuturum & Shrub & Fruit juice & Sore throat & Oral & 38 & 0.53 & 72 & 1.01 \\
\hline & & & & & Gastrointestinal pain & & & & & \\
\hline & & & & & Remove gall stone & & & & & \\
\hline
\end{tabular}


Table 2 Medicinal plants used to treat various diseases in Skardu valley (Continued)

\begin{tabular}{|c|c|c|c|c|c|c|c|c|c|c|}
\hline \multirow[t]{3}{*}{ Brassicaceae } & Lepidium Iatifolium L. SK 115 & Chunma & Herb & Leaves infusion & Liver disease & Oral & 10 & 0.14 & 23 & 0.32 \\
\hline & & & & Root powder & Kidney disease & Oral with water & & & & \\
\hline & & & & & Stomachache & & & & & \\
\hline \multirow[t]{2}{*}{ Campanulaceae } & Codonopsis clematidae C.B. Clarke SK 116 & Bajo mindoq & Herb & Flower infusion & Stress relief & Oral & 11 & 0.15 & 27 & 0.38 \\
\hline & & & & & Memory retention & & & & & \\
\hline \multirow[t]{5}{*}{ Capparidaceae } & Capparis spinosa L. SK 117 & Shorot & Shrub & Fruit juice & Joint pain & Oral & 24 & 0.33 & 104 & 1.46 \\
\hline & & & & & Diuretic & & & & & \\
\hline & & & & & Dropsy & & & & & \\
\hline & & & & Root decoction & Anemia & Oral & & & & \\
\hline & & & & & Kidney disinfectants & & & & & \\
\hline \multirow[t]{5}{*}{ Chenopodiaceae } & Chenopodium album L. SK 118 & Snew & Herb & Leaves infusion & Rheumatism & Oral & 17 & 0.23 & 32 & 0.45 \\
\hline & & & & Leaves poultice & Swollen feet & Topical & & & & \\
\hline & & & & & Sunstroke & & & & & \\
\hline & & & & & Sunburn & & & & & \\
\hline & & & & & Freckles & & & & & \\
\hline \multirow[t]{5}{*}{ Chenopodiaceae } & Chenopodium botrys L. SK 119 & Khama & Herb & Whole plant infusion & Stomachache & Oral & 18 & 0.25 & 17 & 0.23 \\
\hline & & & & & Liver complaints & & & & & \\
\hline & & & & & Headache & & & & & \\
\hline & & & & & Laxative & & & & & \\
\hline & & & & & Diuretic & & & & & \\
\hline \multirow[t]{3}{*}{ Crassulaceae } & Rhodiola imbricata Edgew. SK 120 & Chundol & Herb & Root powder & Cough, fever & Oral with milk & 11 & 0.15 & 38 & 0.53 \\
\hline & & & & & Headache & & & & & \\
\hline & & & & & Anemia & & & & & \\
\hline \multirow[t]{3}{*}{ Cupressaceae } & Juniperus excelsa M. Bieb. SK 121 & Hlashuk & Shrub & Fruit juice & Remove kidney stone & Oral & 37 & 0.52 & 66 & 0.92 \\
\hline & & & & & Rheumatism & & & & & \\
\hline & & & & & $\begin{array}{l}\text { Respiratory disorders } \\
\text { backache }\end{array}$ & & & & & \\
\hline \multirow[t]{4}{*}{ Elaeagnaceae } & Hippophae rhamnoides L. SK 122 & Choq & Shrub & Fruit juice & Cough & Oral & 65 & 0.91 & 117 & 1.64 \\
\hline & & & & & Arthritic pain & & & & & \\
\hline & & & & & Burns & & & & & \\
\hline & & & & & Eczema & & & & & \\
\hline \multirow[t]{2}{*}{ Ephedraceae } & Ephedra gerardiana Wall. SK 123 & Chay & Shrub & Stem decoction & Rheumatism & Oral & 31 & 0.43 & 98 & 1.38 \\
\hline & & & & & Syphilis & & & & & \\
\hline
\end{tabular}


Table 2 Medicinal plants used to treat various diseases in Skardu valley (Continued)

\begin{tabular}{|c|c|c|c|c|c|c|c|c|c|c|}
\hline & & & & Fruit juice & Respiratory disorders & Oral & & & & \\
\hline & & & & & $\begin{array}{l}\text { Asthma/breathing } \\
\text { problem }\end{array}$ & & & & & \\
\hline & & & & & Tonic & & & & & \\
\hline \multirow[t]{4}{*}{ Gentianaceae } & Swertia petiolata Royle SK 124 & Brama & Herb & Root paste & Opthalmia & Topical & 15 & 0.21 & 33 & 0.46 \\
\hline & & & & Leaves decoction & Scleritis & Oral & & & & \\
\hline & & & & & Stomach Inflammation & & & & & \\
\hline & & & & & Liver tonic & & & & & \\
\hline Gentianaceae & Gentiana olivieri Griseb. SK 125 & Tikta & Herb & Fresh leaf & Diabetes & Oral & 22 & 0.30 & 16 & 0.22 \\
\hline \multirow[t]{5}{*}{ Geraniaceae } & Geranium nepalense Sweet SK 126 & Bamik & Herb & Fruit juice & Renal disease & Oral & 44 & 0.61 & 43 & 0.60 \\
\hline & & & & Root poultice & Rheumatic pain & Topical & & & & \\
\hline & & & & & Toothache & & & & & \\
\hline & & & & & Wounds & & & & & \\
\hline & & & & & Cuts & & & & & \\
\hline Lamiaceae & $\begin{array}{l}\text { Dracocephalum nuristanicum Rech. f. \& Edelb. } \\
\text { SK } 106\end{array}$ & Shamdun & Herb & Seed infusion & $\begin{array}{l}\text { Gastrointestinal } \\
\text { disorders }\end{array}$ & Oral & 41 & 0.57 & 74 & 1.04 \\
\hline \multirow[t]{2}{*}{ Lamiaceae } & Mentha royleana Wall. ex Benth SK 127 & Foling & Herb & Leaves infusion & Dysentery & Oral & 26 & 0.36 & 41 & 0.57 \\
\hline & & & & & Abdominal pain & & & & & \\
\hline \multirow[t]{4}{*}{ Lamiaceae } & Perovskia abrotanoides Kar. SK 128 & Faring bursay & Subshrub & Flower infusion & Expel worms & Oral & 45 & 0.63 & 69 & 0.97 \\
\hline & & & & & Leishmaniasis & & & & & \\
\hline & & & & & Rheumatic pain & & & & & \\
\hline & & & & & Tonic & & & & & \\
\hline \multirow[t]{2}{*}{ Lamiaceae } & Nepeta leucolaena Benth. ex Hook.f. K 129 & Askuta & Herb & Leaves decoction & Stomach problem & Oral & 40 & 0.56 & 53 & 0.74 \\
\hline & & & & & Constipation & & & & & \\
\hline \multirow[t]{3}{*}{ Lamiaceae } & Thymus linearis Benth. SK 130 & Tumburu & Herb & Whole plant infusion & Pneumonia & Oral & 42 & 0.59 & 57 & 0.80 \\
\hline & & & & & Cough, cold & & & & & \\
\hline & & & & & Respiratory disorders & & & & & \\
\hline \multirow[t]{4}{*}{ Lamiaceae } & Prunella vulgaris L. SK 131 & Harswa & Herb & Leaves decoction & Stomachache & Oral & 30 & 0.42 & 24 & 0.33 \\
\hline & & & & & Sore throat & & & & & \\
\hline & & & & & Diuretic & & & & & \\
\hline & & & & & Tonic & & & & & \\
\hline \multirow[t]{2}{*}{ Ericaceae } & Rhododendron anthopogon D. Don. SK 132 & Chauman & Shrub & Leaves infusion & $\begin{array}{l}\text { Cough, cold lung } \\
\text { disorders }\end{array}$ & Oral & 36 & 0.50 & 55 & 0.77 \\
\hline & & & & Flower juice & Inflammations & & & & & \\
\hline
\end{tabular}


Table 2 Medicinal plants used to treat various diseases in Skardu valley (Continued)

\begin{tabular}{|c|c|c|c|c|c|c|c|c|c|c|}
\hline \multirow[t]{4}{*}{ Onagraceae } & \multirow[t]{4}{*}{ Epilobium latifolium L. SK 133} & \multirow[t]{4}{*}{ Pondol } & \multirow[t]{4}{*}{ Herb } & Flower paste & Itching pimples & Topical & 37 & 0.52 & 28 & 0.39 \\
\hline & & & & \multirow[t]{3}{*}{ Leaves decoction } & Skin problems & \multirow[t]{3}{*}{ Oral } & & & & \\
\hline & & & & & Antidote & & & & & \\
\hline & & & & & Febrifuge & & & & & \\
\hline \multirow[t]{2}{*}{ Papilionaceae } & \multirow[t]{2}{*}{ Astragalus psilocentros Fisch SK 134} & \multirow[t]{2}{*}{ Sokhrus } & \multirow[t]{2}{*}{ Subshrub } & \multirow[t]{2}{*}{ Root Leaves infusion } & Teeth cleaning & \multirow[t]{2}{*}{ Oral } & \multirow[t]{2}{*}{51} & \multirow[t]{2}{*}{0.71} & \multirow[t]{2}{*}{81} & \multirow[t]{2}{*}{1.14} \\
\hline & & & & & Stomach problems & & & & & \\
\hline Papilionaceae & Sophora mollis Graham SK 135 & Khakhul & Shrub & Seed paste & Hepatitis & Topical & 61 & 0.85 & 90 & 1.26 \\
\hline \multirow[t]{5}{*}{ Plantaginaceae } & \multirow[t]{5}{*}{ Plantago lanceolata L. SK 136} & \multirow[t]{5}{*}{ Sman hrswa } & \multirow[t]{5}{*}{ Herb } & \multirow[t]{2}{*}{ Flower infusion } & Diarrhea & \multirow[t]{2}{*}{ Oral } & \multirow[t]{5}{*}{29} & \multirow[t]{5}{*}{0.40} & \multirow[t]{5}{*}{48} & \multirow[t]{5}{*}{0.67} \\
\hline & & & & & Dysentery & & & & & \\
\hline & & & & \multirow[t]{3}{*}{ Leaves decoction } & $\begin{array}{l}\text { Asthma/breathing } \\
\text { problem }\end{array}$ & \multirow[t]{3}{*}{ Oral } & & & & \\
\hline & & & & & Bronchitis & & & & & \\
\hline & & & & & Gastritis & & & & & \\
\hline Polygonaceae & Bistorta amplexicaulis (D.Don.) Greene & Onbu & Herb & Root infusion & Sore throat & Oral & 21 & 0.29 & 24 & 0.33 \\
\hline & SK $13 /$ & & & & Laryngitis & & & & & \\
\hline & & & & & Tonic & & & & & \\
\hline Polygonaceae & Oxyria digyna (L.) Hill. SK 138 & Span harswa & Herb & Leaves decoction & Jaundice & Oral & 27 & 0.38 & 21 & 0.29 \\
\hline & & & & & Dysentery & & & & & \\
\hline & & & & & Gastritis & & & & & \\
\hline Polygonaceae & Polygonum affine D. Don. SK 139 & Strin mindoq & Herb & Root decoction & Lung disorder & Oral & 23 & 0.32 & 38 & 0.53 \\
\hline & & & & Flower infusion & Fever, flu & Oral & & & & \\
\hline & & & & & Expel worms & & & & & \\
\hline Polygonaceae & Rheum australe D. Don SK 140 & Lachu & Herb & Leaves and stem infusion & Hepatitis B & Oral & 22 & 0.30 & 34 & 0.47 \\
\hline & & & & & Appendicitis & & & & & \\
\hline & & & & & Dysentery & & & & & \\
\hline & & & & & Constipation & & & & & \\
\hline & & & & Root poultice & Swellings & Topical & & & & \\
\hline & & & & & Burns & & & & & \\
\hline Primulaceae & Primula denticulata Smith SK 141 & Daoo & Herb & Leaves infusion & Diabetes & Oral & 28 & 0.39 & 44 & 0.61 \\
\hline & & & & & Urinary ailments & & & & & \\
\hline & & & & Root poultice & Opthalmia & Topical & & & & \\
\hline & & & & & Wound healing & & & & & \\
\hline Ranunculaceae & $\begin{array}{l}\text { Aconitum violaceum Jacquem. ex Stapf } \\
\text { SK } 142\end{array}$ & Booma & Herb & Root decoction & Leprosy & Oral & 34 & 0.47 & 48 & 0.67 \\
\hline
\end{tabular}


Table 2 Medicinal plants used to treat various diseases in Skardu valley (Continued)

\begin{tabular}{|c|c|c|c|c|c|c|c|c|c|c|}
\hline & & & & & $\begin{array}{l}\text { Asthma/breathing } \\
\text { problem }\end{array}$ & & & & & \\
\hline & & & & & Snake bite & & & & & \\
\hline & & & & & Antidote febrifuge & & & & & \\
\hline \multirow[t]{2}{*}{ Ranunculaceae } & Caltha alba Jacquemc SK 143 & Pilling & Herb & Root paste & Toothache & Topical & 32 & 0.45 & 40 & 0.56 \\
\hline & & & & & Muscular pain & & & & & \\
\hline \multirow[t]{4}{*}{ Ranunculaceae } & Delphinium brunonianum Royle SK 144 & Makhoting & Herb & Aerial parts infusion & Pneumonia & Oral & 38 & 0.53 & 49 & 0.69 \\
\hline & & & & & Headache & & & & & \\
\hline & & & & & Stomachache & & & & & \\
\hline & & & & Seed poultice & Healing of injuries & Topical & & & & \\
\hline Rosaceae & $\begin{array}{l}\text { Potentilla argyrophylla Wall. ex Lehm. var. } \\
\text { atrosanguinea (G.Lodd. ) Hook.f. SK } 145\end{array}$ & Serfo harswa & Herb & Whole plant paste & Wounds & Topical & 16 & 0.22 & 18 & 0.25 \\
\hline Rosaceae & Potentilla bifurca L. SK 146 & Tarqan & Herb & Flower infusion & Diarrhea & Oral & 39 & 0.54 & 26 & 0.36 \\
\hline \multirow[t]{3}{*}{ Rosaceae } & \multirow[t]{3}{*}{ Potentilla salesoviana Steph. SK 152} & \multirow{3}{*}{$\begin{array}{l}\text { Karfo } \\
\text { mindoq }\end{array}$} & \multirow[t]{3}{*}{ Herb } & \multirow[t]{3}{*}{ Flower infusion } & Stomachache & \multirow[t]{3}{*}{ Oral } & \multirow[t]{3}{*}{10} & \multirow[t]{3}{*}{0.14} & \multirow[t]{3}{*}{17} & \multirow[t]{3}{*}{0.23} \\
\hline & & & & & Fever & & & & & \\
\hline & & & & & Cough, cold & & & & & \\
\hline Rosaceae & Rosa brunonii Lindl. SK 147 & Siya & Shrub & Bark infusion & Blood purification & Oral & 63 & 0.88 & 105 & 1.47 \\
\hline \multirow[t]{5}{*}{ Saxifragaceae } & \multirow{5}{*}{$\begin{array}{l}\text { Bergenia stracheyi (Hook. f. \& Thorns.) Engl. } \\
\text { SK } 148\end{array}$} & \multirow[t]{5}{*}{ Khichlay } & \multirow[t]{5}{*}{ Herb } & \multirow[t]{2}{*}{ Leaves infusion } & Stomachache & \multirow[t]{2}{*}{ Oral } & \multirow[t]{5}{*}{44} & \multirow[t]{5}{*}{0.61} & \multirow[t]{5}{*}{52} & \multirow[t]{5}{*}{0.73} \\
\hline & & & & & Diuretic & & & & & \\
\hline & & & & \multirow[t]{3}{*}{ Root poultice } & Tonic & \multirow[t]{3}{*}{ Topical } & & & & \\
\hline & & & & & Opthalmia & & & & & \\
\hline & & & & & Wounds, cuts & & & & & \\
\hline \multirow[t]{5}{*}{ Saxifragaceae } & \multirow[t]{5}{*}{ Bergenia ciliata (Haw.) Sternb. SK 149} & \multirow[t]{5}{*}{ Shaphus } & \multirow[t]{5}{*}{ Herb } & \multirow[t]{2}{*}{ Leaves juice } & Diarrhea & Oral & 41 & 0.57 & 35 & 0.49 \\
\hline & & & & & $\begin{array}{l}\text { Asthma/breathing } \\
\text { problem }\end{array}$ & & & & & \\
\hline & & & & Seed paste & Urinary problems & Topical & & & & \\
\hline & & & & & Opthalmia & & & & & \\
\hline & & & & & Boils & & & & & \\
\hline Scrophulariaceae & Pedicularis cheilanthifolia Schrenk SK 150 & Serfo & Herb & Leaves decoction & Stomachache & Oral & 14 & 0.19 & 26 & 0.36 \\
\hline & & spanthing & & & Tonic & & & & & \\
\hline Scrophulariaceae & Pedicularis pectinatiformis Bonati SK 151 & Sunpo & Herb & Leaves infusion & Body ache & Oral & 12 & 0.16 & 15 & 0.21 \\
\hline & & spanthing & & & Sedative & & & & & \\
\hline
\end{tabular}




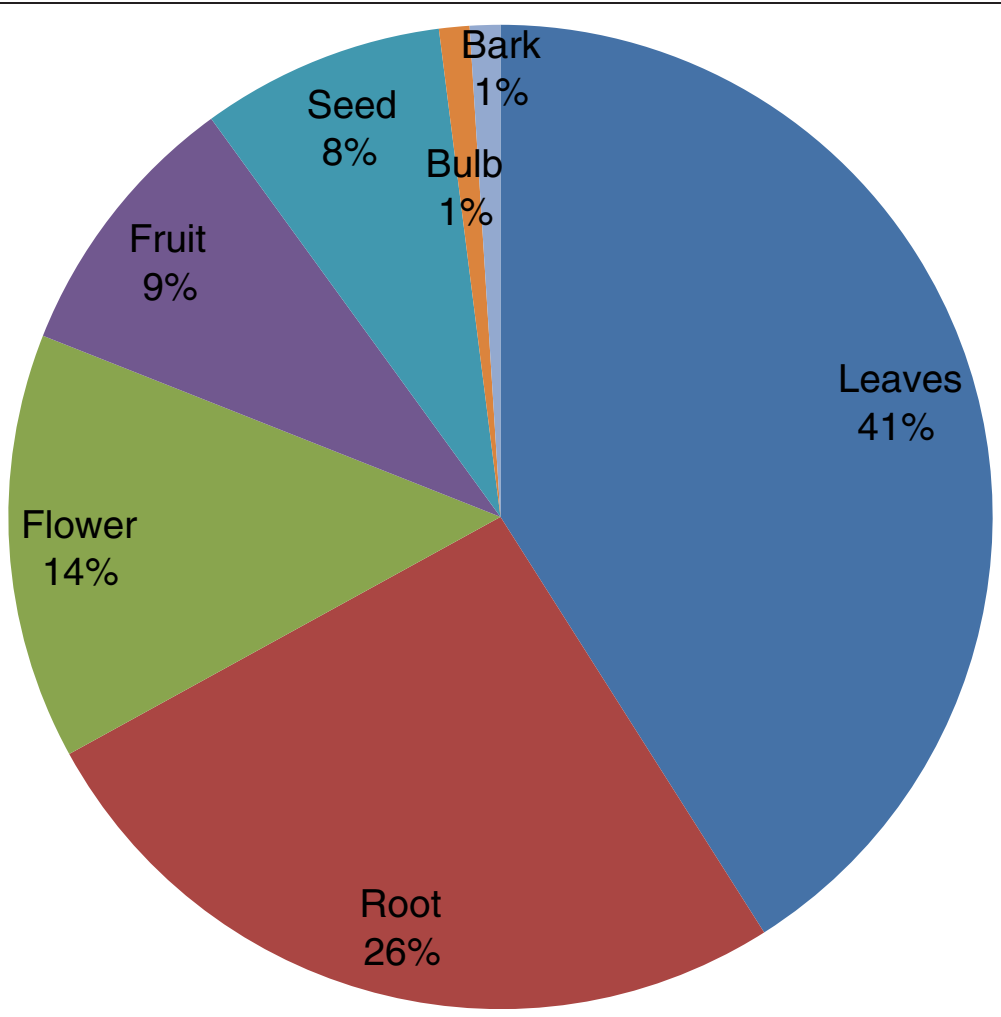

Figure 2 Percentage of plant parts used in herbal preparations.

correspond to the fact that the plants were reported by highest number of informants and RFC directly depends on the number of informants mentioning use of this plant (FC). Figure 6 shows the 9 most popular medicinal plants with highest use value reported by the informants. As shown in Figure 6, Hippophae rhamnoides has the highest use value (1.64) followed by Rosa brunonii (1.47) Capparis spinosa (1.46). The fruit of Hippophae rhamnoides plant is extensively used as a cure in arthritic pain, cough, relieve skin inflammation in eczema and a remedy for heart problems, ulcer, jaundice and urinary disorders. However, some additional uses of this species were also reported in previous ethnobotanical studies in other parts of Pakistan [37]. Hippophae rhamnoidesas the characteristic and dominant species of the Skardu valley has an important role in constructing human-nature interrelationship in the area. Local people living in this valley have used

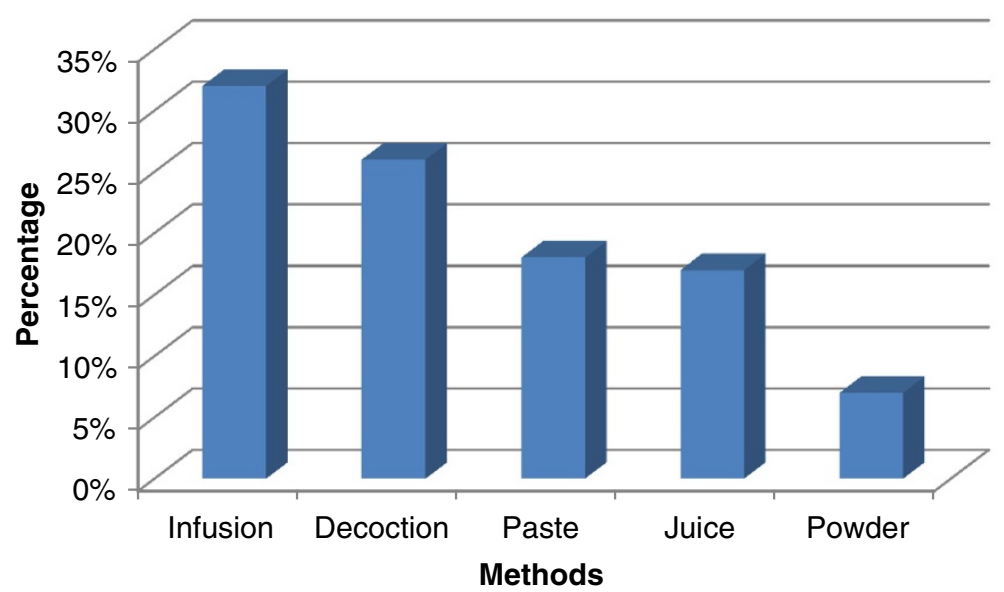

Figure 3 Preparation methods of herbal medicine. 


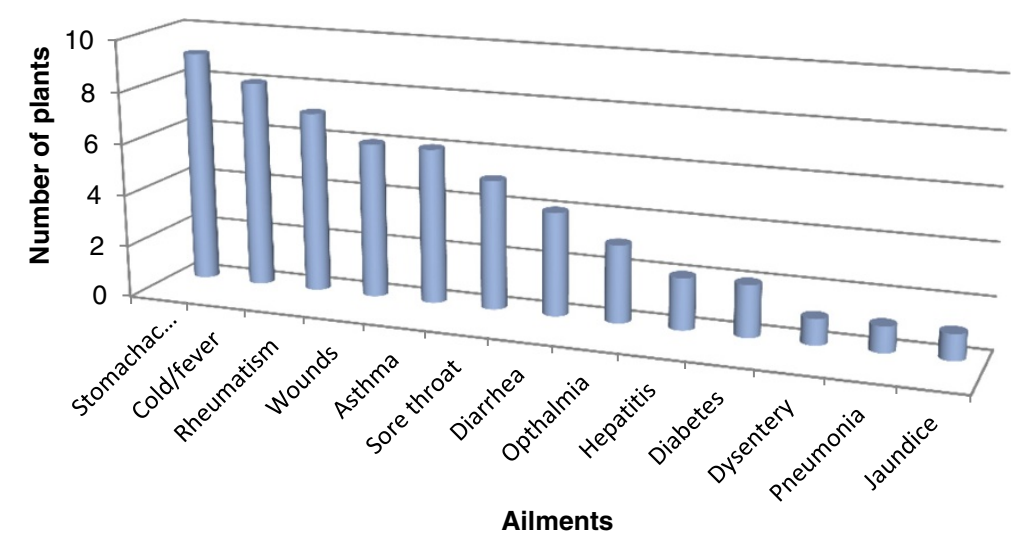

Figure 4 Number of plants used to treat various diseases.

different parts of this plant for various purposes of medicinal, fuel wood, household making, ornamental and decorative purposes. There is no study as such conducted by previous workers, particularly in the Skardu valley from our region, in which the RFC and UV values are calculated. The articles in which the cultural index (CI) is calculated were examined, it is seen that there is a clear difference in the type of most cited species, for example, in the study by Abbasi et al. [38] Ficus carica and Ficus palmata were the most cited species with the highest (CI) as compared to our report species Hippophae rhamnoides and Rosa burnonii. RFC and UV values obtained from the reported species indicate the degree of indigenous knowledge shared by local people regarding the use of medicinal plants in the treatment of the various ailments. These recorded values of RFC and UV were found to be higher in case of some important medicinal species, which could be attributed to the trend of utilization of herbal drugs in the valley.

The value of RFC ranges from 9 percent to 91 percent in the medicinal use of plants/herbs. The former is linked to Jurinea Dolomiaea from the family Asteraceae while the latter is associated with Hippophae Rhamnoides from family Elaeagnaceae. However, on average, the relative frequency citation is 39 percent. Likewise the UV of medicinal plants ranges from 0.12 to 1.64 which shows least relative importance of Jurinea Dolomiaea from the family Asteraceae to the highest importance for Hippophae Rhamnoides from family Elaeagnaceae. These findings are consistent with that from RFC. Keeping these configurations in view, it can be itemized that 64.39 percent inhabitants of the study area are dependent for medication upon these indigenous plants.

The Pearson correlation coefficient between RFC and UV was 0.732 with P-value less than 1 percent, which

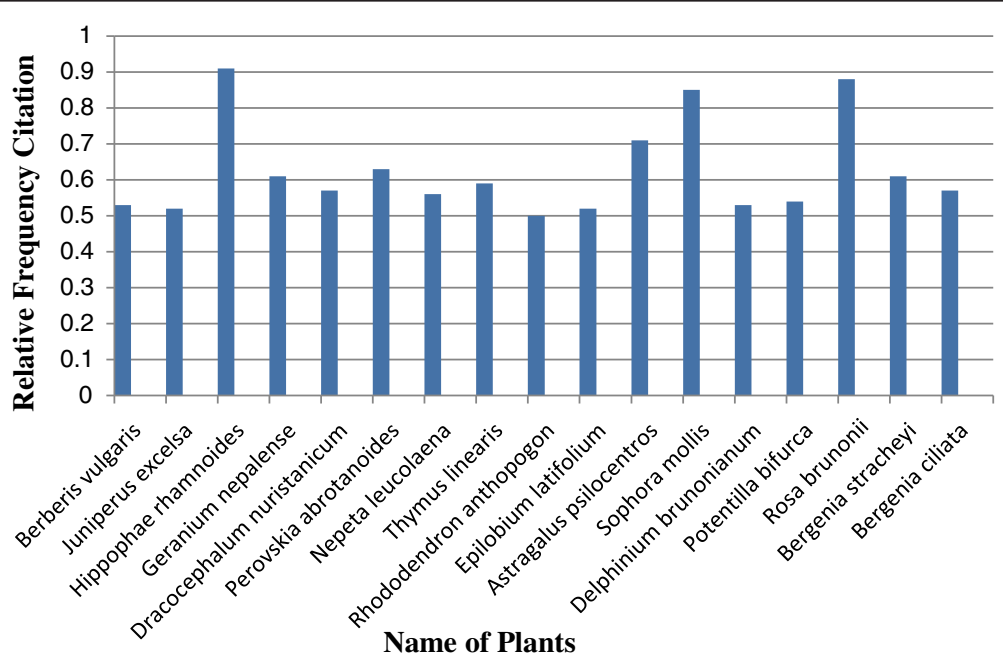

Figure 5 Plant species with highest Relative Frequency of Citation (RFC). 


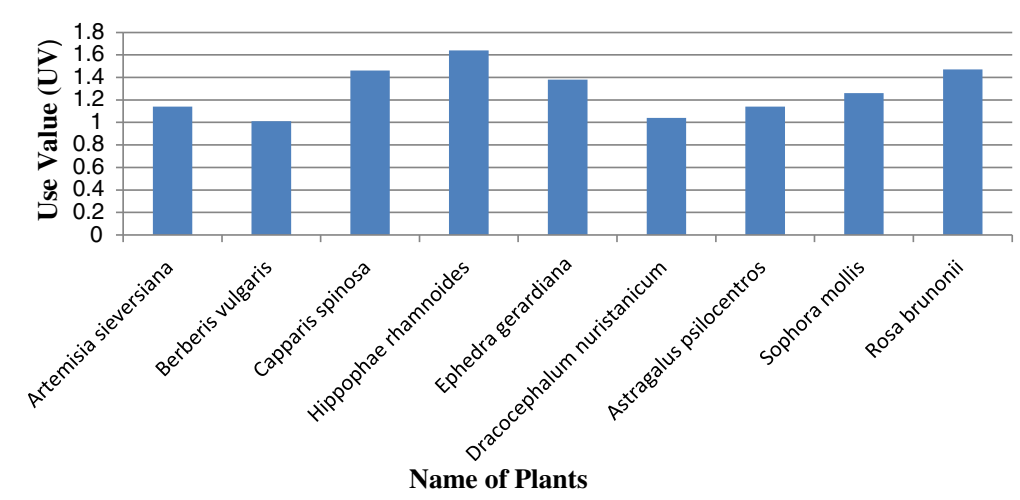

Figure 6 Plant species with highest Use Value (UV).

provide the evidence of a high positive significant association between the local importance of each species and relative importance of the use of plants. It implies that more use of species of the informants tend to increase the number of usable medicinal plants. The fact that RFC and UV are strongly positively correlated means that their patterns across species match. However, some species may have high RFC and UV while others have low values. The degree to which RFC and UV varies across species was measured numerically by $\mathrm{r}^{2}$ which states that around 54 percent variation in RFC can be explained by that of UV. Thus, the findings imply the empirical robustness among these two indices (Table 3). These findings are further supported by a graph drawn in the shape of the scattered plot which reflects a strong positive relationship between RFC and UV (Figure 7).

\section{Comparative analysis with ethnobotanical literature}

In comparative analysis of ethnomedicinal use of plants in Skardu valley and its neighboring districts, the rest of Pakistan, neighboring countries and similar studies at world level, eight published studies which meet scientific ethnobotanical standards were used. This comparison shows that there is a high similarity index between our study and ethnobotanical studies of $[39,40]$ which is in agreement that Asteraceae (7-species), Lamiaceae (6-species), Rosaceae (4-species) and Polygonaceae (4-species) are

Table 3 Summary Stats for Relative Frequency Citation (RFC) and Use Value (UV)

\begin{tabular}{lllll}
\hline & Mean & Standard deviation & Minimum & Maximum \\
\hline RFC & .3944 & .20452 & .09 & .91 \\
UV & .6406 & .37059 & .12 & 1.64
\end{tabular}

Association between RFC and UV by using Pearson Correlation Method

r $\quad 0.732(0.000)^{*}$

$r^{2} \quad 0.536$

*Figure in parenthesis is P-values for significance of correlation coefficient. the dominantly represented families, herbaceous life form being in highest percentage and leaves are the highly consumed part of plants. This similarity may be attributed to the similar flora, cultural uses and close proximity of the study areas but the species like Hippophae rhamnoides, Rosa brunonii, Capparis spinosa, Ephedra gerardiana, Sophora mollis, Artemisia sieversiana, Astragalus psilocentros, Berberis vulgaris and Dracocephalum nuristanicum with high UV does not agrees which may be due to specificity between regions. In contrast, the study of [41] in Serbian district Zlatibor which is not in close proximity, but confirms our findings on the high similarity index of family representation, life forms and part used.

Similar to our study $[36,39,40,42]$ shows that the Asteraceae and Rosaceae have frequently represented families in the study areas.

The studies conducted in the surrounding districts and neighboring countries show the lowest indices of similarity [36,42-45] which probably could be attributed to cultural loss of ethnobotanical knowledge, change in floral diversity, leading to the fact that in each area people develop a use of the available species [46]. Quantitative ethnobotany calculations are performed, allowing us to discuss the results and their novelty value compared with other studies. This analysis shows a novelty of ethnomedicinal information suggesting further investigations into the ethnomedicinal wealth of the study area Table 1 .

\section{Threats to medicinal plants and indigenous knowledge in} Skardu Valley

Skardu valley is one of the naturally enriched regions of high mountains in the Karakorum- Himalayan ranges that make it unique with traditional cultural heritage but equally challenging to its community. The low standard of living and scattered population in high terrains consequently inaccessible or minimized the modern healthcare facilities for the majority of the population. This is 


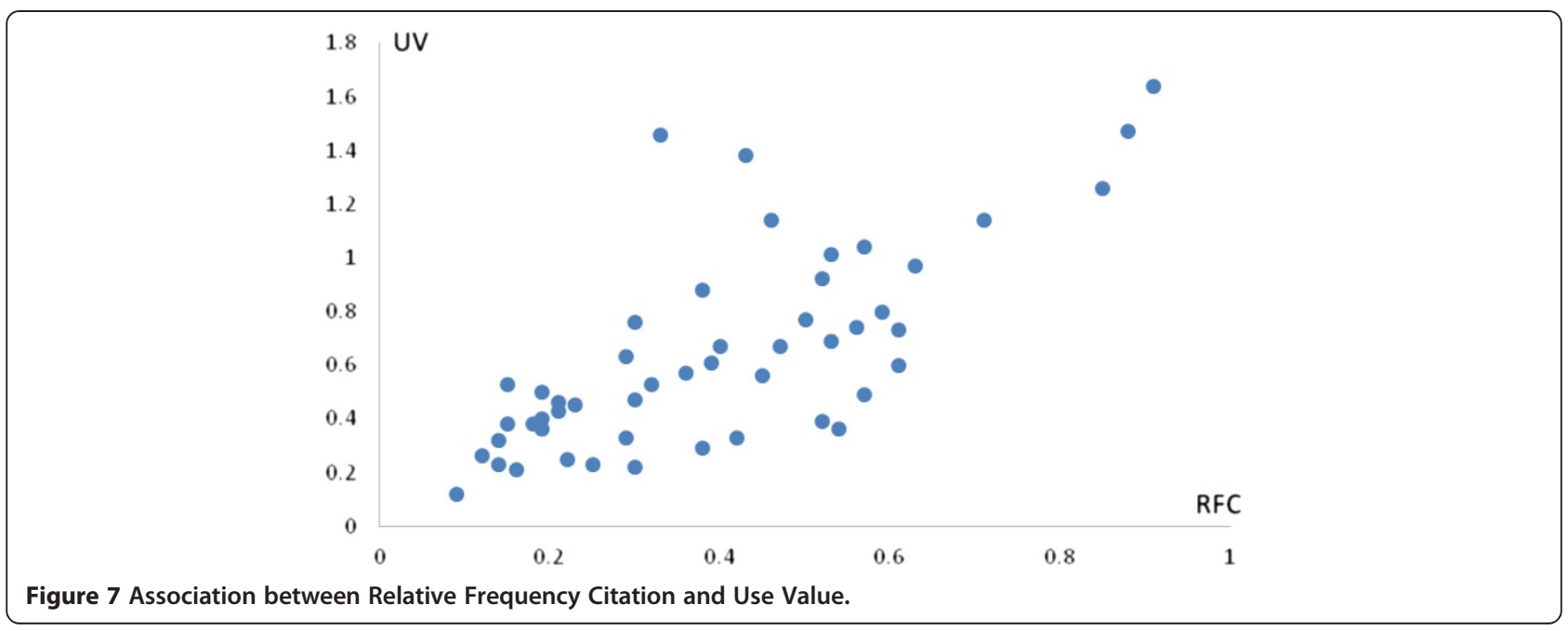

the main reason behind the dependency of local people on medicinal plant resources to treat common day ailments. Besides the Karakoram Highway (KKH), the sole connection of Gilgit-Baltistan with the rest of Pakistan is often blocked due to the frequent land sliding round the year and intense snowfall in the winter which makes it isolated physically. It forces the local community to rely on native biodiversity to meet their daily needs. The people of the valley largely depend on wild plants for fuel, food supplements, medicine, construction material, nutrients and livestock feed. The majority of the people is engaged in agriculture, animal husbandry and forests related works. The vegetation in the valley is mostly inferior scrubs while herbs are abundant in alpine and subalpine pastures. The flora of fragile alpine meadows has been overexploited for traditional medicine because medicinal plant collectors invariably uproot the entire plant and due to this, the regrowth of some very important medicinal plants is retarded [37,47]. There is a dramatic degradation of habitat due to collection of fuel wood and shrubs to meet the domestic energy requirement as the temperature drops below $-25^{\circ} \mathrm{C}$ in winter (NovemberFebruary) and there is no alternate source. The ruthless use of valuable medicinal plants of the grazing animals is indeed a great injustice. Pastures and rangelands are used for livestock herding by the local communities on a periodic basis like high pastures are used in summer and low rangelands in autumn. During the course of study, the informants were selected randomly from the tribal communities based on their rich knowledge with long experience in utilization of medicinal plants and most of the informants belonged to or above 60 years age. In this survey, we observed that the wealth of knowledge is rapidly vanishing due to the death of elderly rural people. Due to this, transfer of indigenous knowledge from generation to generation is now endangered in this area and tends towards disappearance. Preservation of this stock of knowledge is highly important for the socioeconomic prosperity of the region. On one hand, the community can be motivated to be a party of conserving their precious resources while on the other hand experts engaged in the policy making can realize to make the flora of this region in the lime light.

\section{Conclusion}

This study reports the first quantitative ethnomedicinal survey in the nine selected sites of Skardu valley of Northern Pakistan. Among 50 plant species belonging to 25 reported families, Asteraceae and Lamiaceae are the most used families in the area. The leaves are the favored part of local users. The most treated illnesses of the Skardu valley using medicinal plants are grouped into 33 pathological disorders. The most popular medicinal plants of the valley known by the local communities includes Hippophae rhamnoides, Rosa brunonii, Capparis spinosa, Ephedra gerardiana, Sophora mollis, Artemisia sieversiana, Astragalus psilocentros, Berberis vulgaris and Dracocephalum nuristanicum based on their highest UV and RFC values. The Pearson correlation coefficient between RFC and UV was 0.732 with P-value less than 1 percent, which provide the evidence of a high positive significant association between the local importance of each species and relative importance of the use of plants. In this way, we have compiled significant baseline data regarding indigenous knowledge about the native medicinal plants for treating common ailments is now ready to be further investigated phytochemically and pharmacologically which leads to natural drug discovery and development. Meanwhile, the medicinal plant flora of Skardu valley are threatened by major factors such as habitat degradation, grazing, expansion of new agricultural lands, and unsustainable picking of herbal plants to generate income. 
Practical steps should be taken immediately to ensure the inclusion of relevant flora within conservation designations for sustainable use. This would be meant to enhance the welfare level of the people living in the Skardu valley in particular and other ecosystems of similar nature in general.

\section{Competing interests}

The authors declare that they have no competing interests.

\section{Authors' contributions}

$\mathrm{AB}$ : This work is part of my PhD thesis; I carried out ethnomedicinal survey in highly remote areas of Pakistan-Skardu valley. MA and MZ supervised this ethnobotanical project and helped in plant identification. MA and SS helped in editing the manuscript and quantitative data analysis. MAA and TBH provided technical expertise in compiling data into the draft. AS, MAA and MA helps in statistics. All the authors read and approved the final manuscript.

\section{Acknowledgements}

We are very thankful to the support provided by Zulfiqar Ali (Army officer) during field surveys in very tough and complex sites of Skardu valley. We are also grateful to Zahid Ali for his immense help and cooperation in the collection of plants and data in local language. The authors are grateful to the local informants who provided their valuable information and Higher Education Commission (HEC) Pakistan. This research is supported by High Impact Research MoE Grant UM.C/625/1/HIR/MoE/SC/04 from the Ministry of Education Malaysia. Thanks also to UMRG (RG257-13AFR) and FRGS (FP038-2013B) for their support.

\section{Author details}

'Department of Plant Sciences, Quaid-i-Azam University, Islamabad 45320, Pakistan. ${ }^{2}$ School of Chemical Engineering, Universiti Sains Malaysia, Nibong Tebal 14300, Malaysia. ${ }^{3}$ Laboratoire de Chimie des Matériaux, Faculté des Sciences, Université Mohammed Premier, Oujda 60000, Morocco. ${ }^{4}$ Department of Agricultural Economics, PMAS-Arid Agriculture University, Rawalpindi, Pakistan. ${ }^{5}$ Department of Botany, PMAS-Arid Agriculture University, Rawalpindi, Pakistan. ${ }^{6}$ Department of Geology, University of Malaya, 50603 Kuala Lumpur, Malaysia.

Received: 25 September 2013 Accepted: 9 April 2014 Published: 9 May 2014

\section{References}

1. Hocking GM: Pakistan medicinal plants 1. Q Planta Mater Vegetation 1958 9:103-119.

2. Ibrar M, Hussain F, Sultan A: Ethnobotanical studies on plant resources of ranyal hills, district shangla Pakistan. Pak J Bot 2007, 39:329-337.

3. Malcolm JR, Liu C, Miller LB, Allnutt T, Hansen L: Habitats at risk: global warming and species loss in globally significant terrestrial ecosystems. Gland, Switzerland: WWF Fund for Nature; 2002.

4. Pei SJ: Mountain Culture and Forest Resource Management of Himalaya In Himalayan Ecosystem. Edited by Tewari DW. Dehra Dun, India: Intel Book Distribution; 1992:114-304.

5. Hussain F, Khaliq A: Ethnobotanical studies on some plants of Dabargai Hills. NARC, Islamabad: Swat. In Proceedings of First Training Workshop on Ethnobotany and its Application to Conservation; 1996:207-215.

6. Pie SJ, Manadhar NP: Source of some local medicines in the Himalayan Regions. Ecosystems: Himalayan; 1987:77-112.

7. Stewart RR: An Annotated Catalogue of the Vascular Plants of West Pakistan and Kashmir. Karachi, Pakistan: Fakhri Printing Press; 1972.

8. Nasir E, Ali Sl: Flora of Pakistan. Islamabad/ Karachi, Fasc: National Herbarium, NARC/Department of Botany, University of Karachi; 1970-2002. No. 1-207.

9. Ali SI, Qaiser M: A Phyto-geographical analysis of the phanerogams of Pakistan and Kashmir. Proc R Soc Edinb Biol 1986, 89B:89-101.

10. Alam J: Endemic Flora of Gilgit and Baltistan and Conservation Strategies for Threatened Endemic Taxa. Karachi: PhD thesis, Department of Botany, University of Karachi; 2010.

11. Woods CA, Kilpatrick CW, Rafiq M, Shah M, Khan W: Biodiversity and Conservation of the Deosai Plateau, Northern Areas, Pakistan. In Biodiversity of Pakistan. Edited by Mufti SA, Woods CA, Hasan SA. Islamabad Pakistan: Pakistan Museum of Natural History; 1997:33-61.
12. Sultana K, Shah M, Upson TM: Altitudinal distribution of Grasses, Sedges and Rushes of Deosai Plateau. Elec J Env Agricult Food Chem 2007, 6:2517-2525.

13. Shaheen H, Qureshi RA: Vegetation types of sheosar lake and surrounding landscape in deosai plains of North Pakistan, Western Himalayas. J Med Plants Res 2011, 5:599-603.

14. Shinwari ZK, Gilani SS: Sustainable harvest of Medicinal Plants at Bulashbar Nullah, Astore (Northern Pakistan). J Ethnopharmacol 2003, 84:289-298.

15. Qureshi RA, Ghufran MA, Sultana KN, Ashraf M, Khan AG: Ethnobotanical studies of Medicinal plants of gilgit district and surrounding areas. Ethnobotany Res Appl 2006, 5:115-122.

16. Khan SW, Khatoon S: Ethnobotanical studies on useful trees and shrubs of haramosh and bugrote valleys, in gilgit Northern Areas of Pakistan. Pak J Bot 2007, 39:699-710.

17. Khan SW, Khatoon S: Ethnobotanical studies on useful herbs of haramosh and bugrote valleys, in Gilgit Northern Areas of Pakistan. Pak J Bot 2008, 40:43-58.

18. Hussain I, Bano A, Ullah F: Traditional drug therapies from various medicinal plants of central karakoram national park, Gilgit-Baltistan Pakistan. Pak J Bot 2011, 43:79-84.

19. Shedayi AA, Gulshan B: Ethnomedicinal uses of plant resources in gilgitbaltistan of Pakistan. J Med Plants Res 2012, 6:4540-4549.

20. Fahad S, Bano A: Ethnobotanical and physiological studies of some endangered plant species collected from two different altitudes in Gilgit Baltistan. Pak J Bot 2012, 44:165-170.

21. Ahmad M, Bano A, Zafar M, Khan MA, Chaudhary MJl, Sultana S: Pollen morphology of some species of the family asteraceae from the alpine zone, Deosai Plateau, northern Pakistan. Palynology 2013, 37(2):189-195.

22. Jamal Z, Ahmad M, Zafar M, Sultana S, Khan MA, Shah GM: Medicinal plants used in traditional folk recipes by the local communities of Kaghan valley, Mansehra, Pakistan. Indian J Tradit Knowl 2012, 11:634-639.

23. Vitalini S, Iriti M, Puricelli C, Ciuchi D, Segale A, Fico G: Traditional knowledge on medicinal and food plants used in Val San Giacomo (Sondrio, Italy)-an alpine ethnobotanical study. J Ethnopharmacol 2013, 145:517-529.

24. Tardio J, Pardo-de-Santayana M: Cultural importance indices: a comparative analysis based on the useful wild plants of Southern Cantabria (Northern Spain). Econ Bot 2008, 62:24-39.

25. Gujrati D: Essentials of econometrics. 2nd edition. Irwin: McGraw-Hill; 1999:56.

26. Walpole RE: Introduction to statistics. 3rd edition. Prentice Hall: Prentice Hall PTR; 1982:378-382

27. Koop G: Analysis of economic data. 3rd edition. John Wiley \& Sons Ltd; 2009:34-36.

28. Bano A, Ahmad M, Khan MA, Zafar M, Sultana S, Ullah Z: Pollen morphology of four endemic species of Pedicularis L. from alpine zone of the Deosai Plateau, Himalayan range. Bangl J Plant Taxon 2012, 19(1):1-5.

29. Cornara L, La Rocca A, Marsili S, Mariotti MG: Traditional uses of plants in the Eastern Riviera (Liguria, Italy). J Ethnopharmacol 2009, 125:16-30.

30. Benı'tez G, MR G' I-T, Molero-Mesa J: Pharmaceutical ethno-botany in the western part of Granada province (southern Spain): ethnopharmacological synthesis. J Ethnopharmacol 2010, 129:87-105.

31. Blanco E, Macia MJ, Morales R: Medicinal and veterinary plants of EI Caurel (Galicia, northwest Spain). J Ethnopharmacol 1999, 65:113-124.

32. Ayyanar M, Ignacimuthu S: Traditional knowledge of kani tribals in Kouthalai of Tirunelveli hills, Tamil Nadu, India. J Ethnopharmacol 2005, 102:246-255.

33. Scherrer AM, Motti R, Weckerle CS: Traditional plant use in the area of monte vesole and ascea, cilento national park (Campania, southern Italy). J Ethnopharmacol 2005, 97:129-143

34. Giday M, Asfaw Z, Elmqvist T, Woldu Z: An ethnobotanical study of medicinal plants used by the Zay people in Ethiopia. J Ethnopharmacol 2003, 85:43-52.

35. Macia MJ, Garcia E, Vidaurre PJ: An ethnobotanical survey of medicinal plants commercialized in the markets of La Paz and El Alto, Bolivia. J Ethnopharmacol 2005, 97:337-350.

36. Wazir SM, Dasti AA, Shah J: Common medicinal plants of chapursan valley, Gojal II, Gilgit- Pakistan. I Res (Science) Bahauddin Zakariya University, Multan, Pakistan 2004, 15:41-43.

37. Khan B, Abdukadir A, Qureshi R, Mustafa G: Medicinal uses of plants by the inhabitants of Khunjerab National Park, Gilgit Pakistan. Pak J Bot 2011, 43:2301-2310. 
38. Abbasi AM, Mir AK, Munir HS, Mohammad MS, Mushtaq A: Ethnobotanical appraisal and cultural values of medicinally important wild edible vegetables of Lesser Himalayas- Pakistan. Ethnobiol Ethnomed 2013, 9:84.

39. Gorsi MS, Mairaj S: Ethnomedicinal survey of plants of khanabad village and its allied areas, District Gilgit. Asian J Plant Sci 2002, 1(5):604-615.

40. Abbas Q, Qureshi R, Naqvi AUN, Khan SN, Hussain I: Floristic inventory and ethnobotanical study of the naltar valley (Karakorum ranges), Gilgit Pakistan. Pak J Bot 2013, 45:269-277.

41. Salvikin K, Zdunic G, Menkovic N, Zivkovic J, Cujic N, Terescenko M, Bigovic D: Ethnobotanical study on traditional use of medicinal plants in south-western Serbia, Zlatibor district. J Ethnopharmacol 2013, 146:803-810.

42. Abbasi AM, Khan MA, Zafar M: Ethnomedicinal assessment of some selected wild edible fruits and vegetables of lesser Himalayas, Pakistan. J Ethnopharmacol 2013, 45:215-222.

43. Kadir MF, Sayeed MSB, Mia MMK: Ethnopharmacological survey of medicinal plants used by the indigenous and tribal people in Rangamati Bangladesh. J Ethnopharmacol 2012, 144:627-637.

44. Shah MG, Khan MA: Common medicinal folk recipes of siran valley, Mansehra, Pakistan. Ethnobotanical leaflets 2006, 10:49-62.

45. Khan ZS, Khuroo AA, Dar GH: Ethnomedicinal survey of Uri, Kashmir Himalaya. Indian J Tradit Knowl 2004, 3(4):351-357.

46. Savo V, Caneva G, Guarrera PM, Reedy D: Folk phytotherapy of the amalfi coast (Campania, Southern Italy). J Ethnopharmacol 2011, 135:376-392.

47. Khan I, Razzaq Islam M: Ethnobotanical studies of some medicinal and aromatic plants at higher altitudes of Pakistan. Am Eurasian J Agric Environ Sci 2007, 2:470-473.

doi:10.1186/1746-4269-10-43

Cite this article as: Bano et al:: Quantitative ethnomedicinal study of plants used in the skardu valley at high altitude of Karakoram-

Himalayan range, Pakistan. Journal of Ethnobiology and

Ethnomedicine 2014 10:43.

\section{Submit your next manuscript to BioMed Central and take full advantage of:}

- Convenient online submission

- Thorough peer review

- No space constraints or color figure charges

- Immediate publication on acceptance

- Inclusion in PubMed, CAS, Scopus and Google Scholar

- Research which is freely available for redistribution 